\title{
The significance of nonviable eggs for Daphnia population dynamics
}

\author{
Maarten Boersma ${ }^{1}$ and Jacobus Vijverberg \\ Netherlands Institute of Ecology, Center for Limnology, Rijksstraatweg 6, 3631 AC Nieuwersluis, The Netherlands
}

\begin{abstract}
Egg mortality was studied in populations of Daphnia galeata, Daphnia cucullata, and the hybrid between these species. In Tjeukemeer, a shallow eutrophic lake in the Netherlands, egg mortality in daphnids manifested itself as an apparent increase in the frequency of eggs in the early developmental stages. Close examination of these eggs revealed that many of them stopped their development. Egg mortality was observed during a 1-month period in autumn 1990 and for about 2 months in spring 1991. The percentage of females carrying nondeveloping eggs averaged $20 \%(\mathrm{SD}=11 \%)$ in the 1990 period and $35 \%(\mathrm{SD}=20 \%)$ in spring 1991 , resulting in an average reduction of the birth rate of $0.043(\mathrm{SD}=0.027)$ and $0.040(\mathrm{SD}=0.030)$. The maximum incidence of nondeveloping eggs was as high as $70 \%$. Because it is difficult to recognize nondeveloping eggs, little is known about the cause of this arrested development. Our results suggest that food quantity was not an important factor and that egg mortality was caused by nutritional deficiency of the lake seston in certain pcriods of the year. Egg mortality can have serious consequences for the population development of daphnids, and it might be a more common phenomenon than is supposed. An analysis of zooplankton population dynamics is essential to propcrly assess the viability of eggs, since ignoring egg mortality will cause both birth and death rates to be overestimated.
\end{abstract}

Many studies have focused on the seasonal dynamics of freshwater zooplankton species and the causes of changes in population abundances during the year (e.g. Gliwicz 1985; Lampert 1988). Traditionally, predation and food limitation have received the most attention as factors influencing zooplankton population dynamics. Both can influence demographic rates. Predation by fish or invertebrates acts directly on the mortality rate of the population. In addition, predation can also influence the birth rate via selective predation of either adults or juveniles. Food limitation can also affect both the birth rate, by a decrease in fecundity, and the death rate, as a result of starvation. Egg mortality has thus far received considerably less attention as a factor influencing demographic rates and hence the seasonal dynamics of zooplankton. Egg mortality has been discussed mostly in relation to size-selective predation of egg-bearing adults (e.g. Threlkeld 1979a; Tessier 1984), and only a limited number of studies have reported on nonpredatory sources of egg mortality (c.g. Brooks 1946; Redfield 1981; Threlkeld $1985 a$ ). The paucity of studies is probably due to the difficulty inherent in differentiating between dead and

\footnotetext{
1 Present address: Max-Planck-Institut für Limnologie, PF 165, D-24302 Plön, Germany.

\section{Acknowledgments}

We thank Koos Swart and Steven Visser for collecting the samples, which were counted by Peter Mac Gillavry, Rob Hoekstra, and Chris de Groot. Hans Hoogveld determined the algal densities. Aafje Landman and Theo Frank helped with the laboratory experiments. We acknowledge Ramesh Gulati, Joop Ringelberg, Klaus Schwenk, Pict Spaak, Onno van Tongeren, and Karen Wiltshire for comments on the manuscript.

These investigations were supported by the Life Science Foundation (SLW), which is subsidized by the Netherlands Organization for Scientific Research (NWO).
}

living eggs. Even fewer attempts have been made to estimatc the impact of egg mortality on zooplankton population dynamics. Threlkeld (1985a) reported that egg abortion was closely linked in time to some cladoceran population declines, suggesting that egg mortality might be important for population development. However, Threlkeld $(1985 \mathrm{~b})$, also stated that he found no consistent change in the frequency of degenerate eggs, which would link egg mortality causally with those declines.

Most researchers who reported on egg mortality found that eggs stopped their development in the early stages, and this was sometimes followed by total lysis (Richman 1958; Hall 1964; Redfield 1981). Hebert (1974) observed a different pattern of cgg mortality in Daphnia magna. Individuals of this species produced eggs that developed to the cmbryo stage with well-developed limbs and a single black eyespot without shedding their first egg membrane.

Little is known about the causes of egg mortality. Most workers have suggested that food conditions (both quantity and quality) are important. Brooks (1946), Richman (1958), and Redfield (1981) suggested that food quantity played a key role. Richman (1958) found egg mortality in Daphnia pulex reared at low Chlamydomonas reinhardtii concentrations and no egg mortality in well-fed animals. Kambestad (unpubl.) observed a correlation between the incidence of egg degeneration in Daphnia longispina and the phytoplankton biomass 1 week earlier, while Brooks (1946) reported that the initial high incidence of degenerated eggs in Daphnia retrocurva disappeared within 2 weeks when animals taken from the field were given an algal-bacteria mixture as food. In a seasonal survey on the occurrence of egg degeneration in the field, however, he found that one of the peak incidences of egg degeneration coincided with the period of maximum egg production of the animals (i.e. with a period when food 


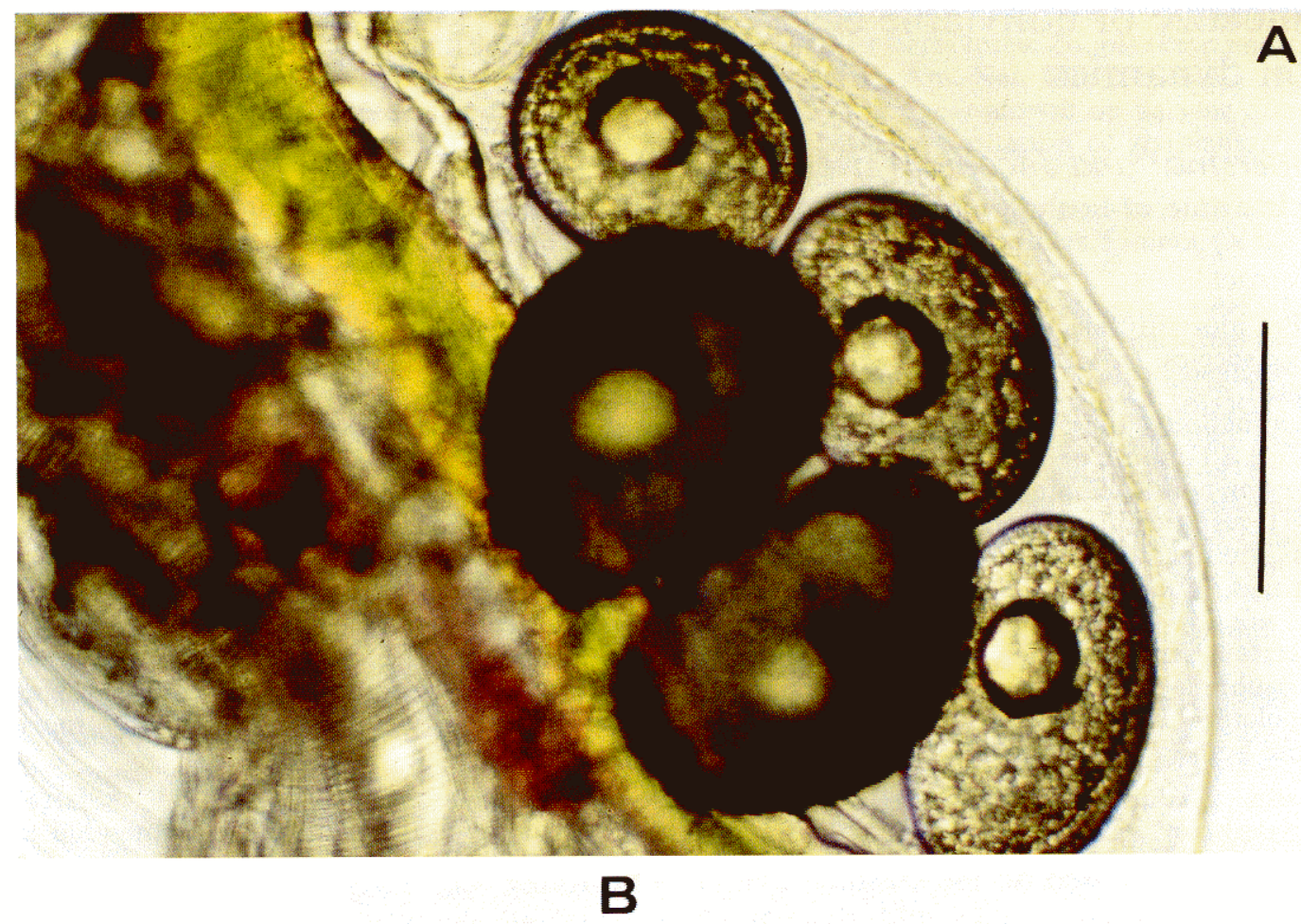

C
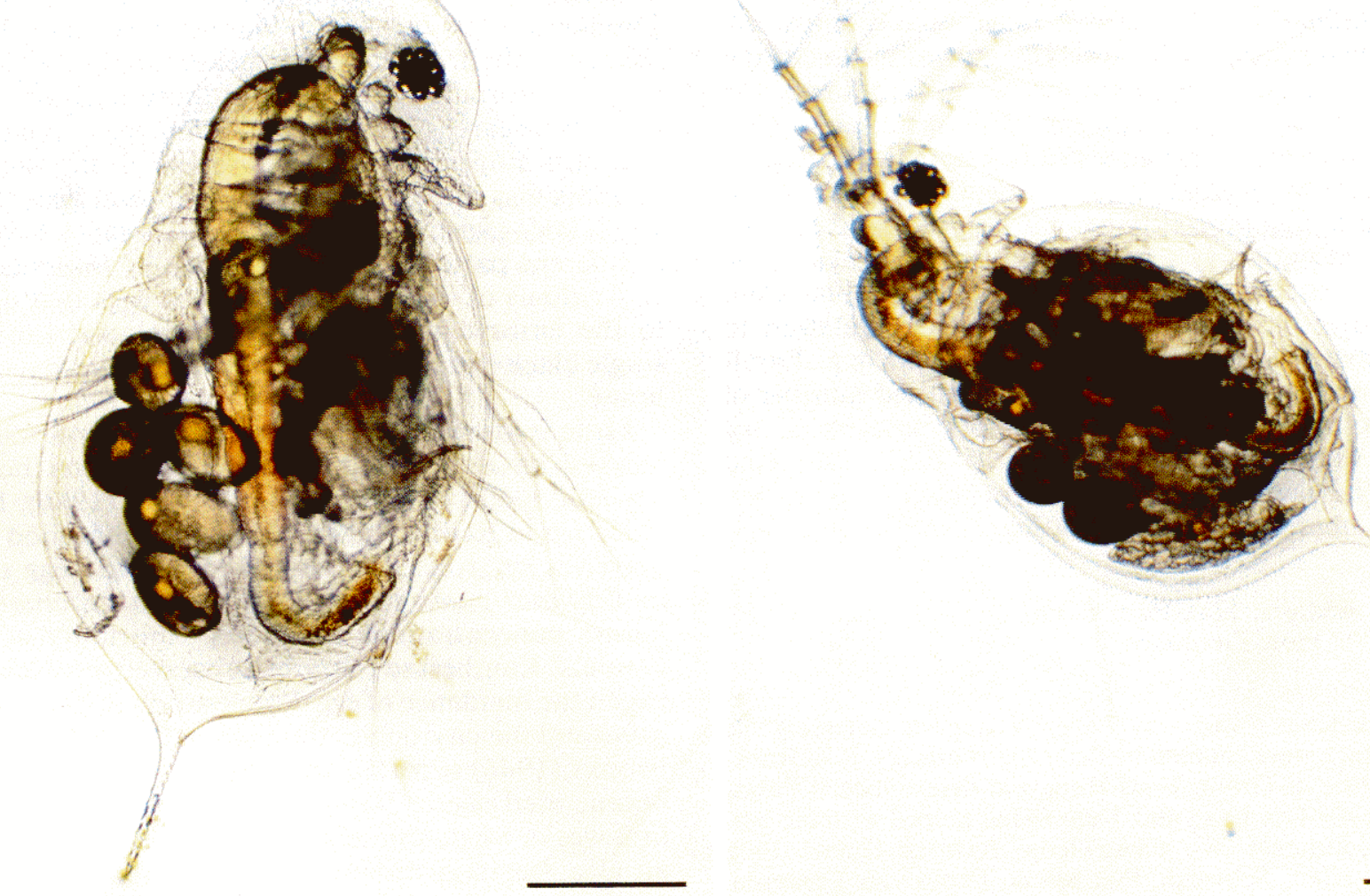
quantity is not likely to be low). Others (Hall 1964; Hebert 1974) have suggested that the effect of food quality might be more important than the effect of food quantity.

Several other factors, such as temperature and parasites, have been suggested to influence egg mortality. Green (1974) reported that one parasite, Blastulidium sp., affects egg development; the effects of temperature were investigated by Orcutt and Porter (1984), who observed in an experiment with Daphnia parvula that the percentage of degenerate eggs increased with temperature. Threlkeld $(1979 b)$, however, did not find a relationship between temperature and egg degeneration in Daphnia pulicaria.

We investigated the possible causes for egg mortality in Daphnia galeata, D. cucullata, and their interspecific hybrid. As food-quality effects were mentioned in the literature, we hypothesized that the quality of the food can be such that egg mortality occurs, but that food quantity is of minor importance. This hypothesis was tested by comparing two different food sources, one a natural lake seston and the other a medium with cultured algae. Because it is difficult to assess the food quantity for daphnids under eutrophic conditions, indirect measurements, such as the average number of eggs, were used to compare the food quantity of the two food sources. We also investigated whether different species or size classes show different patterns in the production of nonviable eggs. Furthermore, a seasonal survey of egg mortality in the field was done to assess the impact of egg mortality on daphnid population dynamics by estimating the influence of egg mortality on population demographic rates.

\section{Materials and methods}

Nondeveloping eggs-Several studies have examined the normal development of parthenogenetic eggs in Daphnia (e.g. Obreshkove and Fraser 1940; Lei and Clifford 1974; Gulbrandsen and Johnsen 1990). At first glance, nondeveloping eggs (NDE) resemble normal eggs (DE) in stage 2A (Gulbrandsen and Johnsen 1990), i.e. the first egg membrane is still intact, the interior of the cells is granulate, and the periphery of the eggs is a little lighter than the center (Fig. 1A). Close examination of these eggs, however, reveals subtle differences in appearance: the eggs are slightly reddish, they have a glassy appearance, and the yolk mass is usually not in the center of the egg (Fig. 1B). In very young egg stages (stage 1 , according to Gulbrandsen and Johnsen 1990), it is impossible to distinguish between developing and nondeveloping eggs. Eggs which are apparently in stage 2 , however, can be successfully classified as NDE or DE. During the whole intermolt period, the appearance of the nondeveloping eggs remains constant. When the female molts, the eggs are shed with the exuviae. In the next instar, females produce new eggs, which either develop normally or are again of the nondeveloping type. Usually, all eggs within a clutch are of the same type, with a low percentage of the clutches containing both types. These mixed clutches might be mistaken as a desynchronization of the egg production cycle with the molt cycle, since at first glance the brood pouch of the animals contains both young eggs and embryos in the last stages of their development (Fig. 1C).

Study site-Tjeukemeer is a shallow eutrophic windexposed lake in the northern part of the Netherlands (Beattie et al. 1979). The lake has a surface area of 21.5 $\mathrm{km}^{2}$ and an average depth of $1.5 \mathrm{~m}$. The littoral zone is poorly developed. As a result of its shallowness in combination with the large influence of the wind, the lake is polymictic, and hence temperature differences within the lake are small. Secchi-disk depth during the summer averages $40 \mathrm{~cm}$. Typically, the algal biomass in Tjeukemeer is dominated by filamentous cyanobacteria, particularly Oscillatoria species (Moed and Hoogveld 1982). In general, there is no clear-water phase.

Laboratory experiments $-D$. galeata, $D$. cucullata, and the hybrid $D$. galeata $\times$ cucullata were cultured in 1990 under two different food conditions: natural Tjeukemeer water filtered through an 80- $\mu \mathrm{m}$ filter (lake seston, LS, medium); and 0.45- $\mu \mathrm{m}$-filtered Tjeukemeer water with Chlamydomonas globosa and Scenedesmus obliquus added at a 1:1 biomass ratio, with a total algal carbon content of $2.5 \mathrm{mg} \mathrm{C}$ liter $^{-1}$ (cultured algae, CA, medium). The algae were cultured axenically in a 2-liter batch culture. To avoid toxicity from inorganic compounds in the medium, we concentrated the algae by centrifuging them twice for $20 \mathrm{~min}$ at $3,000 \mathrm{rpm}$ and washed them with distilled water. After that, the algae were resuspended in $0.45-\mu \mathrm{m}$-filtered Tjeukemeer water.

The experiments were carried out at a light-dark rhythm of $12: 12 \mathrm{~h}$. For each scries, 60 neonates were collected within $12 \mathrm{~h}$ of birth and placed in 100-ml tubes. The clonal composition of the animals in all series within a species was the same and was a mixture of clones present in the field at the time of the experiment and standard clones. All individuals were transferred to clean tubes with fresh medium on alternate days. The number of eggs was counted for every adult instar, the frequency of nondeveloping eggs was established, and the length of the individuals recorded. In order to study the differences in conditions within the growing scason, we carricd out the culture experiment three times: in spring (April-May), summer (July-August), and autumn (October-November) 1990 .

Fig. 1. A. Daphnia galeata $\times$ cucullata with normally developing eggs in stage 2 A (scale bar $=0.1 \mathrm{~mm})$. B. Daphnia cucullata with nondeveloping eggs (scale bar $=0.2 \mathrm{~mm}$ ). C. D. cucullata with a mixed clutch of developing and nondeveloping eggs (scale bar $=0.2 \mathrm{~mm}$ ). 
Table 1. Average number of eggs plus standard deviation (SD) and average size (mm), with SD of the first adult instar of Daphnia galeata, Daphnia galeata $\times$ cucullata, and Daphnia cucullata in the laboratory on LS medium and CA medium in summer 1990, with the number of observations $(N)$.

\begin{tabular}{lccccccc}
\hline \hline & \multicolumn{3}{c}{ LS medium } & & \multicolumn{3}{c}{ CA medium } \\
\cline { 2 - 3 } \cline { 6 - 7 } & Eggs(SD) & Size(SD) & $N$ & & Eggs(SD) & Size(SD) & $N$ \\
\hline D. galeata & $5.9(1.2)$ & $1.46(0.09)$ & 34 & & $4.4(1.7)$ & $1.57(0.09)$ & 47 \\
D. gal $\times$ cuc & $4.3(2.1)$ & $1.14(0.12)$ & 42 & & $3.5(1.7)$ & $1.19(0.07)$ & 46 \\
D. cucullata & $3.1(1.0)$ & $0.86(0.04)$ & 56 & & $3.0(1.0)$ & $0.89(0.07)$ & 49 \\
\hline
\end{tabular}

Field observations-Zooplankton samples were taken every week at five stations in the lake with a Friedinger sampler (a trap-type volume sampler of 5 liters). The cylinder is $60 \mathrm{~cm}$ high, has an i.d. of $10 \mathrm{~cm}$, and is made of Perspex. The samples were pooled, concentrated by passage through a $120-\mu \mathrm{m}$ filter, and preserved in $4 \%$ formaldehyde solution. The animals were counted and measured from the base of the tail spine to the top of the eye to the nearest $0.01 \mathrm{~mm}$. To prevent underestimating fecundity due to egg losses from the brood chamber as a result of the preservative, we took an additional sample with a $120-\mu \mathrm{m}$ plankton net and preserved it in $95 \%$ ethanol. The incidence of degenerate eggs was determined with the Formalin-preserved sample because identifying the viability of the eggs was much easier in those samples.

To assess the food conditions of the zooplankton, we took additional samples for analysis of chlorophyll $a$ and algal biomass. However, because these measures are not reliable indicators of food availability under eutrophic conditions (Boersma and Vijverberg 1994b), we also directly measured the condition of individual $D$. galeata in 1991. Because the length-dependent carbon content is a good measure of the condition of individual zooplankton (Duncan 1985; Duncan et al. 1985; Boersma and Vijverberg 1994b), animals were analyzed for carbon with a UNICARB carbon analyzer, originally developed by Salonen (1979), and linear regression statistics were computed between the logarithm of individual length and the logarithm of the carbon content of thesc animals.

Impact of NDE on demographic rates and population dynamics-From the population development in the field, we computed the actual natural rate of increase of the population, $r_{\text {act }}$ :

$$
r_{\text {act }}=\left(\log N_{t+\Delta t}-\log N_{t}\right) / \Delta t .
$$

$N_{t}$ and $N_{t+\Delta t}$ are the abundances at times $t$ and $t+\Delta t$. The birth rate, $b_{\text {act }}$, can be estimated from the average number of eggs per individual and the temperature-dependent development time of the eggs (Paloheimo 1974):

$$
b_{\text {act }}=\log \left(E_{\text {act }}+1\right) / D,
$$

$E_{\text {act }}$ is the average number of eggs per individual in the population, with NDE accounted for, and $D$ is the egg development time, which was established by culturing the three daphnid taxa at different temperatures (Boersma unpubl.). Naturally, the birth rate would be higher if all eggs were viable, $b_{\text {pot }}$ (the potential birth rate):

$$
b_{\text {pot }}=\log \left(E_{\text {pot }}+1\right) / D .
$$

$E_{\text {pot }}$ is the average number of eggs per individual should all eggs hatch. Comparing birth rates only yields limited information concerning the impact of NDE on the populations since the relative size of the birth rate compared to the population rate of increase, $r$, at the time is also of importancc. Therefore, wc assessed the impact of NDE on the population development as follows: $r_{\text {act }}$ was computed with Eq. 1. As always, the intrinsic rate of increase is the difference between the population birth rate and the death rate, $d$ :

$$
r_{\text {act }}=b_{\text {act }}-d \text {. }
$$

The potential growth rate, $r_{\text {pot }}$, can now be estimated by substituting $b_{\text {pot }}$, the potential birth rate with all eggs viable, for the actual birth rate, $b_{\text {act }}$, under the assumption that the death rate is unaffected by this increase in birth rate:

$$
r_{\mathrm{pot}}=b_{\mathrm{pot}}-d .
$$

Naturally, $r_{\text {act }}=r_{\text {pot }}$ when the percentage of females with $\mathrm{NDE}$ is zero. From $r_{\text {pot }}$, it is possible to compute the potential population size, $N_{\text {pot}}$, for the different species, with every field observation taken as a new starting point. This method should give a conservative estimate of the potential population development, since cumulative processes only act between sampling dates.

\section{Results}

Laboratory-Nondeveloping eggs were found in the laboratory only in summer 1990 and not in spring and autumn 1990 cultures. Only animals fed natural Tjeukemeer lake seston (LS) produced NDE. No individuals with NDE were observed in any of the animals fed with the CA medium. The timing of the occurrence of NDE in these experiments was in synchrony with the pcriod of NDE incidence in the field. The percentage of females with NDE in summer 1990 cultures averaged $18 \%$ for $D$. galeata, $27 \%$ for D. galeata $\times$ cucullata, and $9 \%$ for $D$. cucullata. For these cultures, we analyzed whether a difference in the number of eggs existed between the food types. All species had a higher number of eggs on the LS medium (Table 1); ANCOVA, with species and food type as the independent variables, length as the covariable, and the square root of the number of eggs produced by the first adult instar as the dependent variable, showed 
Table 2. Summary statistics of the effect of species and clutch type on the number of eggs of the first adult instar of animals cultured in the laboratory in summer 1990. Results shown are of an ANCOVA with species and food type as the independent variables, length as covariable, and the square root of egg number as dependent variable.

\begin{tabular}{lrrrr}
\hline \multicolumn{1}{c}{ Effect } & MS & \multicolumn{1}{c}{ df } & $F$ & \multicolumn{1}{c}{$P$} \\
\hline Species & 3.76 & 2 & 43.0 & $<0.001$ \\
Food type & 8.23 & 1 & 94.2 & $<0.001$ \\
Species $\times$ food & 1.86 & 2 & 21.3 & $<0.001$ \\
Error & 0.09 & 267 & & \\
\hline
\end{tabular}

significant effects for both species and food type and for the interaction (Table 2). Tukey's posthoc comparison, however, showed that the differences between food types were significant only for $D$. galeata and $D$. galeata $\times$ cucullata.

In the LS medium, the number of cggs in the clutch differed depending on whether the eggs were of NDE or of DE type (Table 3). ANCOVA, with species and clutch type as the independent variables, length of the females as covariable, and the square root of clutch size as dependent variable, revealed significant main and interaction effects (Table 4), although Tukey's posthoc comparison showed that the difference between the clutch types in the number of eggs produced was significant only for D. galeata $(P<0.001)$.

A significant effect of clutch type on the length of the animals was also found (Table 4). In order to establish whether a relationship of length of the animals with the occurrence of NDEs could be found, we performed a regression analysis with the length of the animals as the indepcndent variable and the absence or presence of nondeveloping eggs (scored as 0 and 1 respectively) as dependent variable. Because these presence-absence data were obviously not normally distributed, a logit regression approach (McCullagh and Nelder 1983) with a maximum likelihood loss function was chosen. This means that the dependent variable is the logit-transformed, $\log [p /$ $(1-p)]$, value of the probability $p$ of NDE occurring. The regression coefficients for length were significantly $>0$ for all three species (Table 5), indicating that the probability of NDE formation increased with increasing length and hence that larger (=older) females were more likely to produce NDE.
Table 4. Summary statistics of the effect of species and clutch type on the length of the animals and on the number of eggs in the clutch in the laboratory cultures in summer 1990. Results shown are of an ANCOVA with species and clutch type as the independent variables, length as covariable, and the square root of egg number as dependent variable and of an ANOVA with species and clutch type as the independent variables and length of the animals as dependent variable.

\begin{tabular}{llrrr}
\hline \multicolumn{1}{c}{ Effect } & MS & df & \multicolumn{1}{c}{$F$} & \multicolumn{1}{c}{$P$} \\
\hline & ANCOVA & & \\
Species & 2.81 & 2 & 7.7 & $<0.001$ \\
Clutch type & 6.38 & 1 & 17.4 & $<0.001$ \\
Species X clutch type & 2.87 & 2 & 7.8 & $<0.001$ \\
Error & 0.37 & 617 & & \\
& ANOVA & & \\
Species & 9.51 & 2 & 597.5 & $<0.001$ \\
Clutch type & 0.67 & 1 & 42.2 & $<0.001$ \\
Species X clutch type & 0.003 & 2 & 0.2 & 0.836 \\
Error & 0.015 & 668 & & \\
\hline
\end{tabular}

Field-The seasonal changes in population abundances for three Daphnia species in the lake were recorded in 1990 and 1991. In August-September 1990 and in May 1991, we observed animals with NDE (Fig. 2). The occurrence of NDE did not differ temporally between species, and the percentage of animals with NDE was generally similar. Moreover, the highest incidences of NDE were found in those periods of the year when the populations were growing (Fig. 2). The maximum frequency of NDE clutches was $~ 30-40 \%$ in 1990 and $70 \%$ in 1991. The abundances of $D$. cucullata were low when NDE occurred in 1991; hence, a reliable estimate of the percentage of nondeveloping clutches could not be made. The chlorophyll $a$ concentration of Tjeukemeer in 1990 and 1991 was not related to the incidence of NDE, nor did we find a significant relationship with temperature or biomass of different algal groups (Fig. 3).

In 1990, both female length and the numbers of eggs were recorded for developing and nondeveloping clutches. An ANCOVA, with species and clutch type as independent variables, length as covariable, and the square root of the number of eggs as dependent variable, showed that the difference in number of eggs between the clutch type was only marginally significant $\left(F_{2,219}=3.68 ; P=\right.$ 0.06 ). As in the laboratory-cultured animals, individuals

Table 3. Average number of eggs plus standard deviation (SD) and average length (size, SD) in the laboratory of Daphnia galeata, Daphnia galeata $\times$ cucullata, and Daphnia cucullata on LS medium in summer 1990. Differences of animals with NDE clutches and animals with DE clutches are shown with number of observations $(N)$.

\begin{tabular}{lccccccc}
\hline & \multicolumn{3}{c}{ Nondeveloping clutches } & & \multicolumn{3}{c}{ Developing clutches } \\
\cline { 2 - 3 } \cline { 6 - 8 } & Eggs(SD) & Size(SD) & $N$ & & Eggs(SD) & Size(SD) & $N$ \\
\hline D. galeata & $5.9(3.5)$ & $1.70(0.14)$ & 31 & & $7.5(2.5)$ & $1.59(0.14)$ & 182 \\
D. gal $\times$ cuc & $5.0(3.0)$ & $1.33(0.13)$ & 36 & & $5.7(2.8)$ & $1.25(0.13)$ & 143 \\
D. cucullata & $4.7(1.8)$ & $1.07(0.20)$ & 24 & & $4.3(2.1)$ & $0.98(0.10)$ & 258 \\
\hline
\end{tabular}


Table 5. Logit regression coefficients of the incidence of NDE clutches in the LS medium in summer 1990 as the dependent variable and the length of the animals as independent variable. Terms: constant-intercept (with significance); length-magnitude of the regression coefficient with length (with significance); and $\chi^{2}$, df, and $P_{\text {all }}-$ significance of the wholc model.

\begin{tabular}{lcccccccc}
\hline \hline & Constant & $P$ & Length & $P$ & $N$ & $\chi^{2}$ & df & $P_{\text {all }}$ \\
\hline Daphnia galeata & -10.2 & $<0.001$ & 5.1 & $<0.001$ & 213 & 14.1 & 1 & $<0.001$ \\
Daphnia gal $\times$ cuc & -7.6 & $<0.001$ & 4.8 & 0.001 & 179 & 11.3 & 1 & $<0.001$ \\
Daphnia cucullata & -8.6 & $<0.001$ & 6.1 & 0.006 & 282 & 11.7 & 1 & $<0.001$ \\
\hline
\end{tabular}

with DE were smaller than animals with NDE (Table 6). A logit regression showed the same pattern as in the animals cultured in the laboratory: regression coefficients for length were all positive (Table 7), although only in the case of the hybrid was the regression coefficient significantly different from zero. In the 1991 NDE period, we found an effect of clutch type on the condition of the animals: animals with NDE had significantly higher length-specific $\mathrm{C}$ contents than did animals with developing clutches $\left(F_{1355}=12.6 ; P<0.001\right)$.

Figure 4 shows the differences between $b_{\text {act }}$ and $b_{\text {pot }}$. Due to the near absence of $D$. cucullata in 1991, sample sizes of this species were so low that the birth-rate pattern is erratic. The difference between $b_{\text {pot }}$ and $b_{\text {act }}$ can be as high as $0.10 \mathrm{~d}^{-1}$-a reduction in birth rate of up to $50 \%$. When $r_{\mathrm{pot}}$ is used to estimate $N_{\mathrm{pot}}$, the impact of NDE on the population development is usually not large, except in onc case (Fig. 5). In 1990, when the maximum percentage of females with NDE was $\sim 30 \%$ for all species, there would have been only a slight increase in population size for all three species. In 1991, despite NDE incidences as high as $70 \%$, the impact on the hybrid population was low. However, the population of $D$. galeata was affected by the NDE, with the potential population of $\sim 150$ ind. liter ${ }^{-1}$, reduced to a lower level of $\sim 70$ ind. liter $^{-1}$.

\section{Discussion}

Causes of egg mortality-Most previous studies have linked the incidence of NDE with poor food quality or low food quantity. Our results show that in general, there was no significant difference between the number of eggs produced in DE clutches and NDE clutches. The overall condition of the animals in the field was high in spring 1991 at the time NDE occurred (Boersma and Vijverberg $1994 b$ ), with the length-dependent carbon content of animals with NDE clutches still significantly higher than the condition of animals with normal eggs. In the field, we did not find a relation between egg degeneration and temperature, chlorophyll $a$ concentrations, or the biomass of blue-greens and other algal taxa. Moreover, we found a
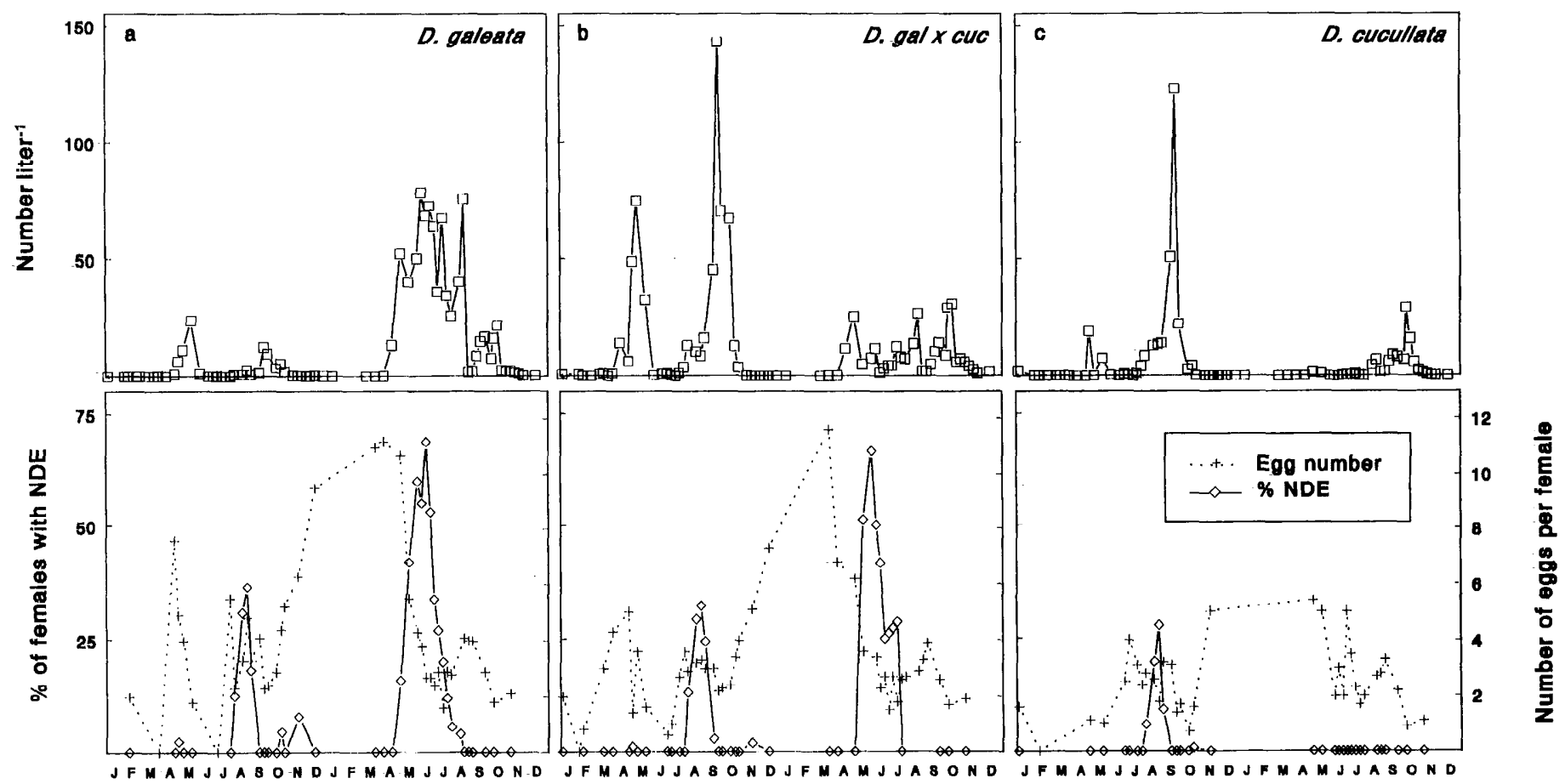

Fig. 2. Seasonal variation in 1990-1991 of population abundances of (a) Daphnia galeata, (b) Daphnia galeata $\times$ cucullata, and (c) Daphnia cucullata (top panels), and of (bottom panels) average number of eggs per adult female and the incidence of NDE. 


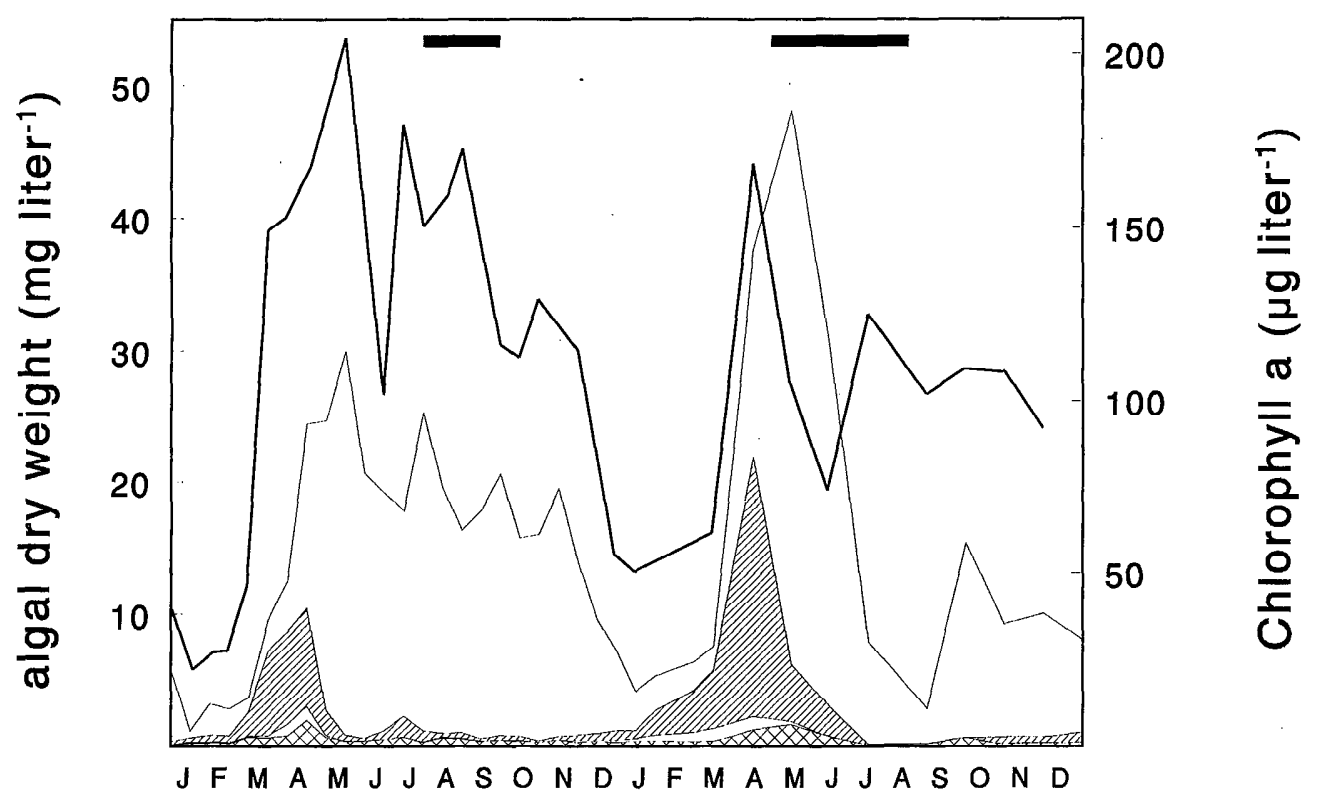

Fig. 3. Seasonal variation in 1990-1991 of the chlorophyll $a$ concentration (solid line) and the biomass of different algal groups: green algae (cross-hatched), cryptophytes (open), diatoms (hatched), and blue-greens (shaded). Horizontal bars indicate the periods of NDE.

nonsignificant relationship of the incidence of NDE with the average number of eggs per sampling date in the field (Kendall's $\tau_{109}=0.009 ; P=0.89$ ).

Kambestad (unpubl.) suggested that the cause of NDE was decreases in food concentration and argued that there might be a time lag between the determination of egg number and the actual collection of the nourishment to produce them; a decline in food availability could mean that daphnids fail to complete the eggs. However, it has been reported that the number of eggs to be produced is determined in the first half of the instar preceding the one that carries the eggs (Bradley et al. 1991). Therefore, it is unlikely that the change in food concentration a week before, as reported by Kambestad (unpubl.), plays a role in the incidence of NDE. More importantly, we analyzed the carbon content of individual DE and NDE. Even when we corrected for the differences in lengths of the mothers, we found that the NDE contained significantly more carbon than the DE contained $\left(F_{1,1335}=12.36 ; P\right.$ $<0.01)$. No NDE were found in the algal-fed cultures, although the animals on this medium had a lower number of eggs (Table 1), indicating lower quantities of food avail- able for the animals in this medium. Moreover, in an experiment with very low food concentrations, we observed no NDE (Boersma and Vijverberg 1994a). Hence, we conclude that the cause of the nondevelopment of eggs should not be sought in low food quantities; most likely, a food deficiency in the natural seston caused eggs to halt their development.

Our laboratory and field results indicate that larger (=older) animals were more likely to produce NDE, since the logit regressions showed that length had a significantly positive regression coefficient on the occurrence of NDE; this result is in contrast to Redfield (1981), who found a higher incidence of NDE in smaller individuals of Daphnia rosea.

Consequences-Overlooking egg mortality can have a serious impact on computing population birth and death rates, resulting in overestimates of both rates. For instance, Brett et al. (1992) found that Paloheimo estimates of death rate overestimated observed death rates by 0.015 - a bias that might have been caused by the occurrence of NDE. Moreover, the occurrence of NDE will

Table 6. Average number of eggs plus standard deviation (SD) and average length (size, SD) in the field in 1990 during the period of NDE occurrence of Daphnia galeata, Daphnia galeata $\times$ cucullata, and Daphnia cucullata. Differences of animals with NDE clutches and animals with DE clutches are shown with number of observations $(N)$.

\begin{tabular}{lccrrrrr}
\hline & \multicolumn{3}{c}{ NDE clutches } & & \multicolumn{3}{c}{ DE clutches } \\
\cline { 2 - 3 } \cline { 6 - 8 } & Eggs(SD) & Size(SD) & $N$ & & Eggs(SD) & Size(SD) & \multicolumn{1}{c}{$N$} \\
\hline D. galeata & $5.2(2.2)$ & $1.38(0.17)$ & 5 & & $3.6(1.4)$ & $1.27(0.09)$ & 23 \\
D. gal $\times$ cuc & $3.5(1.4)$ & $1.13(0.13)$ & 24 & & $3.1(1.3)$ & $1.07(0.12)$ & 104 \\
D. cucullata & $4.1(1.3)$ & $0.94(0.14)$ & 7 & & $3.1(1.5)$ & $0.90(0.09)$ & 63 \\
\hline
\end{tabular}


Table 7. Logit regression coefficients of the incidence of NDE clutches in 1990. Details as in Table 5 .

\begin{tabular}{lcccccccc}
\hline \hline & Constant & $P$ & Length & $P$ & $N$ & $\chi^{2}$ & df & $P_{\text {all }}$ \\
\hline Daphnia galeata & -12.0 & 0.067 & 7.9 & 0.102 & 28 & 3.3 & 1 & 0.069 \\
Daphnia gal $\times$ cuc & -5.8 & 0.007 & 3.9 & 0.039 & 131 & 4.5 & 1 & 0.033 \\
Daphnia cucullata & -6.1 & 0.121 & 4.3 & 0.308 & 70 & 1.1 & 1 & 0.301 \\
\hline
\end{tabular}

lead to an apparent skewness of the egg-age distribution toward younger stages. Hence, biased conclusions may be drawn on the basis of such skewed distributions, e.g. concerning selective feeding of fish on Daphnia with embryos in later developmental stages (Threlkeld 1979a; Tessier 1984; Sed̆a 1992).

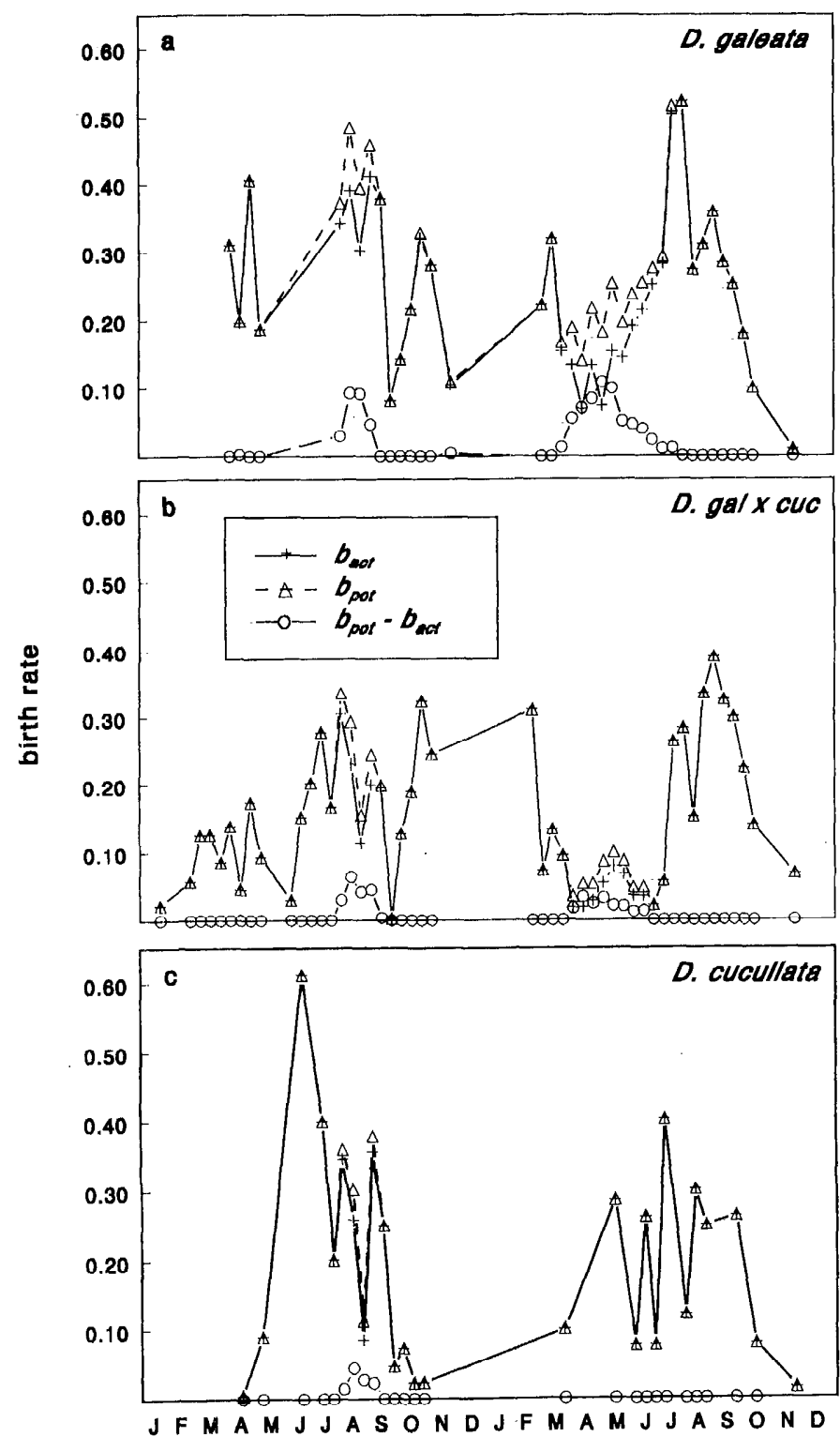

Fig. 4. Seasonal variation in actual birthrate, $b_{\text {act }}$, potential birthrate, $b_{\text {pot }}$, and $b_{\text {pot }}-b_{\text {act }}$ of Daphnia galeata, Daphnia galeata $\times$ cucullata, and Daphnia cucullata.
Because comparing differences in birth rates alone does not yicld complete insight into the impact of NDEs on the population of daphnids, we computed the potential population size if all eggs had been viable. It is unlikely, however, that these population abundances would actually be reached if all of the eggs developed. The most
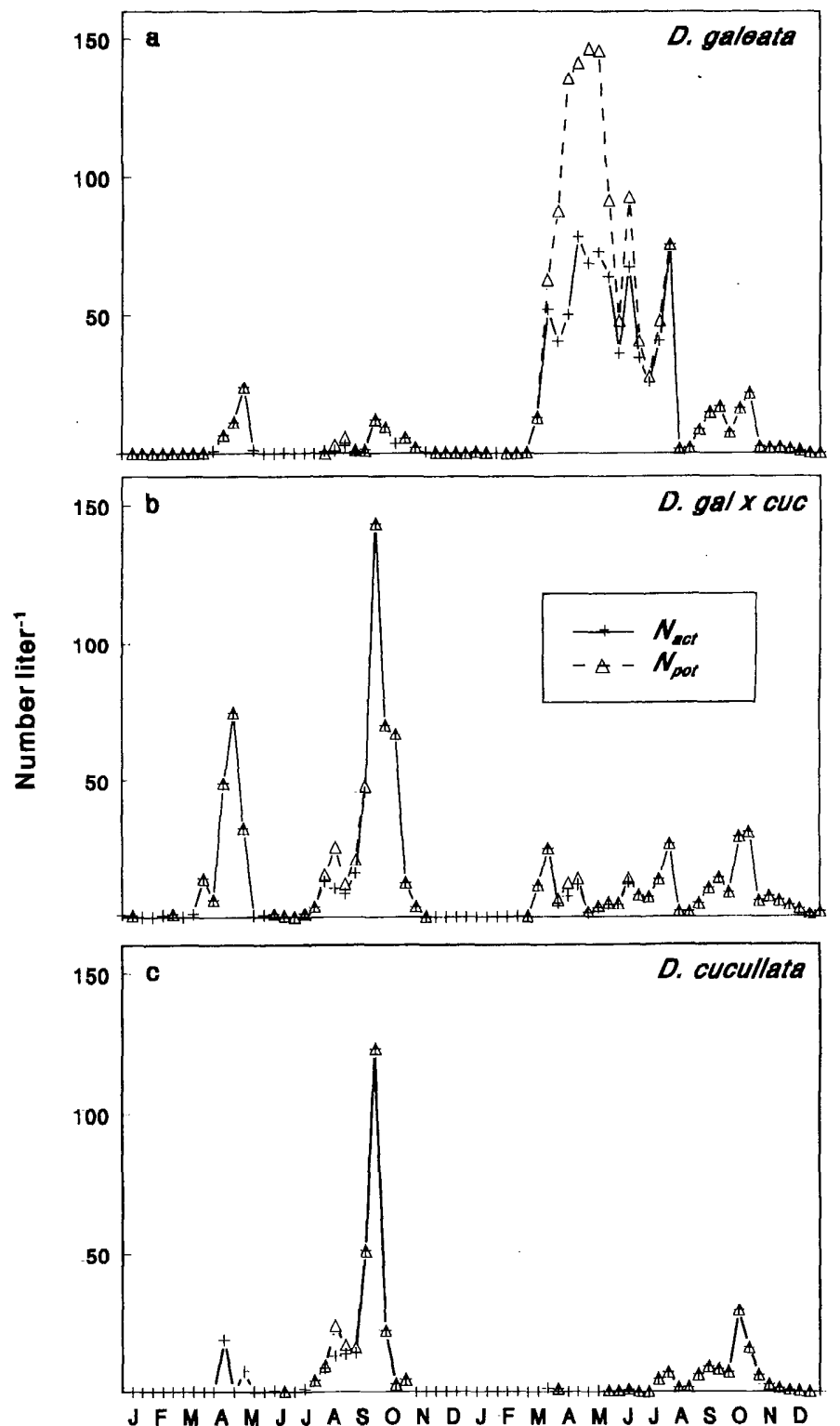

Fig. 5. Seasonal variation in actual population size, $N_{\text {act }}$, and potential population size, $N_{\text {pot }}$, of Daphnia galeata, Daphnia galeata $\times$ cucullata, and Daphnia cucullata. 
important assumption of this computation is that death rates were constant and hence independent of population size. This assumption is not realistic since death rates may increase as a result of food limitation or decrease if the absolute predation pressure is constant. Furthermore, this approach also assumed that the birth rate is unaffected by population abundances, which is not likely to be true either. On the one hand, higher population abundances will probably lead to a lower average number of eggs per female caused by an increase in the level of food limitation; on the other hand, the birth rate will decrease in a growing population anyway becausc of the higher proportion of juveniles in the population. Although $N_{\text {pot }}$ most likely overestimates the real population size if all eggs had been viable, it gives an estimate of the impact of NDE on the population dynamics. Our observation that the larger animals in the population were more likely to produce NDE implies that the effect on the birth rate could be stronger than shown in Fig. 4 because larger animals usually produce more eggs and our computation of birth rates averaged the effect of NDE over all size classes. Moreover, our inability to establish whether eggs in stage 1 are of the NDE type will also lead to an underestimate of the percentage of NDE in the population and, hence, of $N_{\text {por }}$.

It is striking that although frequencies of NDE in $D$. galeata and D. galeata $\times$ cucullata were similar in 1991, the effect on the population differed (Fig. 5). The important factor in the impact of NDE is the magnitude of $r_{\text {act }}$ at the moment NDE are produced. The difference between $b_{\text {act }}$ and $b_{\text {pot }}$ has a small influence on the population when $r$ is negative or a positive value close to zero. Animals born as a result of the increased birth rate under such conditions have a low reproductive value and will die soon. The impact on the population is larger when $r_{\text {act }}$ is positive. In thesc cases, an increase in birth rate will produce new animals with a high reproductive value, and these animals will remain in the population and produce their own offspring. Consequently, the timing of NDE production is important for the effect on the population, with the highest expected effect in growing populations. Hence, it is unlikely that NDE will cause a significant decrease in population numbers, as was suggested by Threlkeld $(1985 a, b)$, unless the period of occurrence is long and the incidence severe. In growing populations, production of NDE could cause a reduction in the increase in population abundances, but this would be difficult to observe in the field.

Threlkeld (1985a) reported considerable temporal differences in egg mortality in five cladoceran species. In this study, we found that the differences in the timing and frequency of NDE between the two Daphnia species and their hybrid were small. This observation could be explained by the fact that $D$. galeata and D. cucullata are closcly related spccics and hence possibly susceptible to the same NDE initiating cues. Consequently, it is difficult to attribute changes in the dominance of different Daphnia species in Tjeukemeer to NDE.

Our conclusion that the production of NDE will lead mainly to a reduction in peak abundances and not much to population declines, combined with the difficulties in detecting NDE in field samples, may be the reason that this phenomenon has generally been overlooked. Even though the causal factors need attention in future studies, we conclude that NDE production can have a profound impact on population development in cladocerans and will certainly lead to overestimates of both birth and death rates.

\section{References}

Beattie, D. M., H. L. Golterman, AND J. ViJVerberg. 1979. An introduction to the limnology of the Friesian lakes. Hydrobiologia 58: 49-64.

BOERSMA, M., AND J. VIJVERBERG. 1994a. Resource depression in Daphnia galeata, Daphnia cucullata, and their interspecific hybrid: Life-history consequences. J. Plankton Res. 16: 1741-1758.

$\longrightarrow$, AND $-1994 b$. Seasonal variations in the condition of two Daphnia species and their hybrid in a eutrophic lake: Evidence for food limitation under field conditions. J. Plankton Res. 16: 1793-1809.

Bradley, M. C., D. J. Baird, AND P. Calow. 1991. Mechanisms of energy allocation to reproduction in the cladoceran Daphnia magna Straus. Biol. J. Linn. Soc. 44: 325-333.

Brett, M. T., L. Martin, AND T. J. KaweCKi. 1992. An experimental test of the egg-ratio method-estimated versus observed death rates. Freshwater Biol. 28: 237-248.

Brooks, J. L. 1946. Cyclomorphosis in Daphnia. 1. An analysis of D. retrocurva and D. galeata. Ecol. Monogr. 16: 409447.

DUNCAN, A. 1985. Body carbon in daphnids as an indicator of the food concentrations available in the field. Ergeb. Limnol. 21: 81-90.

- W. LAMPERT, AND O. RochA. 1985. Carbon weight on length regressions of Daphnia spp. grown at threshold food concentrations. Int. Ver. Theor. Angew. Limnol. Verh. 22: 3109-3115.

Gliwicz, Z. M. 1985. Prcdation or food limitation: An ultimate reason for extinction of planktonic cladoceran species. Ergeb. Limnol. 21: 419-430.

GreEN, J. 1974. Parasites and epibionts of Cladocera. Trans. Zool. Soc. Lond. 32: 417-515.

Gulbrandsen, J., AND G. H. JoHnSEN. 1990. Temperaturedependent development of parthenogenetic embryos in Daphnia pulex de Gecr. J. Plankton Res. 12: 443-453.

HaLl, D. J. 1964. An expcrimental approach to the dynamics of a natural population of Daphnia galeata mendotae. Ecology 45: 94-1 12 .

Hebert, P. D. N. 1974. Ecological differences between genotypes in a natural population of Daphnia magna. Heredity 33: $327-337$.

LAMPERT, W. 1988. The relative importance of food limitation and predation in the seasonal cycle of two Daphnia species. Int. Ver. Theor. Angew. Limnol. Verh. 23: 713-718.

LeI, C. H., AND H. F. ClifFord. 1974. Field and laboratory studies of Daphnia schødleri Sars from a Winterkill Lake of Alberta. Natl. Mus. Can. Publ. Zool. 9: 1-53.

MCCullagh, P., AND J. A. Nelder. 1983. Generalized linear models. Chapman and Hall.

MoED, J. R., AND H. L. HoOGVELD. 1982. The algal periodicity in Tjeukemeer during 1968-1978. Hydrobiologia 95: 223234.

Obreshkove, V., AND A. W. Fraser. 1940. Growth and dif- 
ferentiation in Daphnia magna eggs in vitro. Biol. Bull. 78: 428-436.

Orcutt, J. D., AND K. G. Porter. 1984. The synergistic effects of temperature and food concentration on life history parameters of Daphnia. Oecologia 63: 300-306.

PALOHEIMO, J. E. 1974. Calculation of instantaneous birth rate. Limnol. Occanogr. 19: 692-694.

REDFIELD, G. W. 1981. Nutrition and the degeneration of eggs in a limnetic Daphnia. Int. Ver. Theor. Angew. Limnol. Verh. 21: 1550-1554.

Richman, S. 1958. The transformation of energy by Daphnia pulex. Ecol. Monogr. 28: 273-291.

SALONEN, K. 1979. A versatile method for the rapid and accurate determination of carbon by high temperature combustion. Limnol. Oceanogr. 24: 177-183.

SEĎA, J. 1992. Diurnal periodicity in Daphnia reproduction. Hydrobiologia 246: 119-127.

Tessier, A. J. 1984. Periodicity of egg laying and egg age distributions in planktonic cladocerans. Can. J. Fish. Aquat. Sci. 41: 409-413.

Threlkeld, S. T. 1979a. Estimating cladoceran birth rates: The importance of egg mortality and the egg age distribution. Limnol. Oceanogr. 24: 601-612.

- 1979b. The midsummer dynamics of two Daphnia species in Wintergreen Lake, Michigan. Ecology 60: 165179.

- 1985a. Egg degeneration and mortality in cladoceran populations. Int. Ver. Theor. Angew. Limnol. Verh. 22: 3083-3087.

. $1985 \mathrm{~b}$. Resource variation and the initiation of midsummer declines of cladoceran populations. Ergeb. Limnol. 21: 333-340.

Submitted: 18 November 1993 Accepted: 2 May 1995 Amended: 9 June 1995 